\section{Fatores prognósticos em crianças e adolescentes com Leucemia Linfóide Aguda}

\section{Prognostic factors in children and adolescents with Acute Lymphoblastic Leukemia}

Edinalva Pereira Leite ${ }^{1}$

Maria Tereza Cartaxo Muniz ${ }^{2}$

Alita da Cunha Andrade Cirne de Azevedo ${ }^{3}$

Fernanda Ribeiro Souto 4

Ângela Cristina Lopes Maia 5

Creuza Marilda da Fonseca Gondim ${ }^{6}$

Flávia Miranda Gomes Constantino Bandeira ${ }^{7}$

Raul Antônio Morais Melo ${ }^{8}$

1,2,8 Universidade de Pernambuco. Rua Arnóbio Marques, s. n. Recife, PE, Brasil. CEP: 50.100-130. E-mail: nalvaleite@bol.com.br

3-8 Fundação de Hematologia e Hemoterapia de PernambucoHEMOPE. Recife, PE, Brasil.

\section{Resumo}

Objetivos: descrever características clínico-labora-

Objetives: to describe the clinical and laboratory characteristics, determine rates of response to treatment and pinpoint risk factors that influence the survival of pediatric patients with acute lymphoblastic leukemia (ALL).

Methods: this is a retrospective series of case studies involving 108 patients aged 18 years or under hospitalized for ALL treatment at the Fundação de Hematologia e Hemoterapia de Pernambuco (HEMOPE), Brazil, between January 1993 and December 2001. The following variables were analyzed: gender, age, main symptoms and signs, white blood-cell count, immunophenotype and risk group on diagnosis; rates of remission and relapse, death and overall survival; place of relapse and risk factors for survival. Descriptive measurements were used for the statistical analysis. The patient survival time was estimated using the Kaplan-Meier survival function and Log Rank. The effect of risk factors on survival time was evaluated using the Cox Regression Model.

Results: the results showed a male:female ratio of 1.7:1, a median age of eight years on diagnosis, the frequency of musculoskeletal complaints was $51 \%$, of infiltration of the central nervous system $8 \%$, of ALLPrecursor B $81 \%$ and ALL-T 19\%. The distribution of the groups corresponded to True Basic Risk (12\%), Basic Risk (21\%) and High Risk (67\%). The rates of remission, relapse and overall survival were $86 \%, 24 \%$ and $62.5 \%$, respectively.

Conclusions: the variable having an impact on overall survival was the white blood-cell count. The overall survival rate in the study was influenced by the high frequency of high-risk patients.

Key words Acute lymphoblastic leukemia, Risk factors, Survival toriais, determinar taxas de resposta ao tratamento $e$ identificar fatores de risco que influenciaram na sobrevida de pacientes pediátricos com leucemia linfóide aguda (LLA).

Métodos: estudo retrospectivo do tipo série de casos com 108 pacientes de idade até 18 anos, admitidos para tratamento de LLA na Fundação de Hematologia e Hemoterapia de Pernambuco (HEMOPE), Brasil, de janeiro de 1993 a dezembro de 2001. As variáveis analisadas foram: sexo, idade, principais sintomas e sinais, leucometria, imunofenótipo e grupo de risco ao diagnóstico, taxas de remissão e recaída, óbito e sobrevida global, local de recaída e fatores de risco para a sobrevida. Medidas descritivas foram usadas para a análise estatística. O tempo de sobrevida dos pacientes foi estimado através da função de sobrevida de Kaplan-Meier e Log-Rank. O efeito de fatores de risco no tempo de sobrevida foi avaliado através do Modelo de Regressão de Cox.

Resultados: foi encontrada a relação masculino:feminino de 1,7:1, mediana de idade ao diagnóstico de oito anos, freqüência de queixas músculo-esqueléticas (51\%), infiltração do sistema nervoso central (8\%), LLA-Precursor B (81\%) e LLA-T (19\%). A distribuição dos grupos correspondeu a Risco Básico Verdadeiro (12\%), Risco Básico (21\%) e Alto Risco (67\%). As principais taxas foram: remissão (86\%), óbitos na indução (5,5\%), recaída (24\%) e sobrevida global (62,5\%).

Conclusões: a variável de impacto na sobrevida foi a leucometria. A taxa de sobrevida global foi influenciada pela freqüencia elevada de pacientes considerados de alto risco.

Palavras-chave Leucemia linfoblástica aguda, Fatores de risco, Sobrevivência 


\section{Introdução}

A Leucemia Linfóide Aguda (LLA) é uma neoplasia maligna, caracterizada pelo acúmulo de células linfóides imaturas na medula óssea, sendo os sinais e sintomas apresentados pelos pacientes resultantes de graus variáveis de anemia, neutropenia, trombocitopenia e infiltração dos tecidos por células leucêmicas. ${ }^{1}$

A LLA é a doença maligna mais comum em menores de quinze anos, com pico de incidência entre dois e cinco anos, correspondendo a $25 \%$ de todos os cânceres em crianças brancas nessa faixa etária. ${ }^{2}$ Ela é mais comum em caucasianos quando comparada a afro-americanos com uma relação de 1,5/100.000 na população branca e 0,8/100.000 na população negra. Existe uma maior freqüência em países industrializados e em áreas urbanas, sendo uma doença mais comum no sexo masculino na proporção de 1,3:1..$^{3}$

Embora a causa da LLA seja desconhecida é improvável que a transformação leucêmica seja resultante de evento único, mas sim do acúmulo de múltiplos processos envolvendo interações complexas entre a susceptibilidade do hospedeiro, danos cromossômicos secundários à exposição por agentes químicos ou físicos e à possível incorporação de informações genéticas virais transmitidas às células progenitoras susceptíveis. Anormalidades genéticas hereditárias, tais como Síndrome de Down e Ataxia-telangectasia podem predispor a LLA. ${ }^{1}$ Existem evidências que certos polimorfismos no gene MTHFR, relacionados ao metabolismo do folato, estejam envolvidos na leucemogênese. ${ }^{4}$

A avaliação da incidência dos subtipos de leucemias no mundo tem mostrado variações importantes em relação à distribuição geográfica, sexo, idade e grupos étnicos ou raciais, sugerindo que possam existir diferentes fatores etiológicos. ${ }^{5}$ No Brasil, estudos epidemiológicos mostraram a relação entre subtipos imunológicos de LLA com sazonalidade e condições socioeconômicas, além de evidenciar variações da incidência dessa doença em diferentes estados brasileiros. ${ }^{6,7}$

A chance de cura na LLA tem aumentado e aproxima-se de $80 \%$, sendo este avanço decorrente da melhora no diagnóstico, identificação de fatores prognósticos e utilização de tratamentos adaptados ao grupo de risco de cada paciente. ${ }^{8}$

O objetivo deste trabalho foi caracterizar pacientes pediátricos com LLA do ponto de vista clínico e laboratorial, bem como, determinar fatores que interferiram na sobrevida. Essas informações, além de identificar características locais de apresen- tação dessa patologia, permitem avaliar os resultados do tratamento em instituição pública de referência, contribuindo no conhecimento da assistência à criança com câncer no Brasil.

\section{Métodos}

O estudo foi retrospectivo e do tipo série de casos com 108 pacientes menores de 18 anos de idade, admitidos para tratamento de LLA na Fundação de Hematologia e Hemoterapia de Pernambuco (HEMOPE), em Recife, Pernambuco, Brasil, no período de janeiro de 1993 a dezembro de 2001. A Fundação HEMOPE é um serviço público de referência para tratamento das doenças hematológicas de adultos e crianças maiores de um ano de idade, o que justifica a ausência de casos em crianças abaixo dessa faixa etária. Considerando a existência de três serviços para tratamento de leucemias da infância, os resultados do presente estudo representam à análise de parte dos casos de LLA atendidos no Estado.

Os pacientes foram tratados pelo Protocolo do Grupo Brasileiro para Tratamento da Leucemia Linfóide na Infância (GBTLI-93) ${ }^{9}$ de acordo com três grupos de risco. O Risco Básico Verdadeiro (RBV) incluiu pacientes com idade $\geq$ a 1 ano e $<$ de 10 anos, leucometria inicial $<10.000 / \mathrm{mm}^{3}$, hepato-esplenomegalia inferior a $5 \mathrm{~cm}$ do rebordo costal, ausência de massa mediastinal e de envolvimento do Sistema Nervoso Central (SNC) pela doença. O Risco Básico (RB) incluiu pacientes com idade $\geq$ a 1 ano e $<$ que 10 anos, contagem leucocitária inicial $\geq$ a $10.000 / \mathrm{mm}^{3}$ e $<50.000 / \mathrm{mm}^{3}$, presença de massa mediastinal, fígado e/ou baço com aumento superior a $5 \mathrm{~cm}$ do rebordo costal. Os pacientes foram incluídos no grupo Alto Risco (AR) quando a idade foi $<$ que 1 ano e $\geq$ a 10 anos e/ou contagem de leucócitos $\geq$ a $50.000 / \mathrm{mm}^{3}$ e/ou envolvimento do SNC. Além destes critérios foi recomendado pelo GBTLI-93 ${ }^{9}$ que pacientes com imunofenótipo $\mathrm{T}$ e/ou achados desfavoráveis na citogenética (hipodiploidia, pseudodiploidia, cromossomo Philadelphia) fossem tratados no grupo de AR.

O envolvimento do SNC foi definido pela contagem de leucócitos em líquor $\geq$ a $5 / \mathrm{mm}^{3}$ e demonstração inquestionável de blastos leucêmicos ou pela ocorrência de sinais clínicos, tais como, paralisia de par craniano, síndrome hipotalâmica e evidência de compressão medular.

O planejamento terapêutico do GBTLI-93 ${ }^{9}$ compreende as fases de indução, intensificação, reindução e manutenção (Tabela 1). A radioterapia foi realizada para os pacientes de AR na fase de reindução. 
Protocolo de tratamento da leucemia linfóide aguda do GBTLI-93.

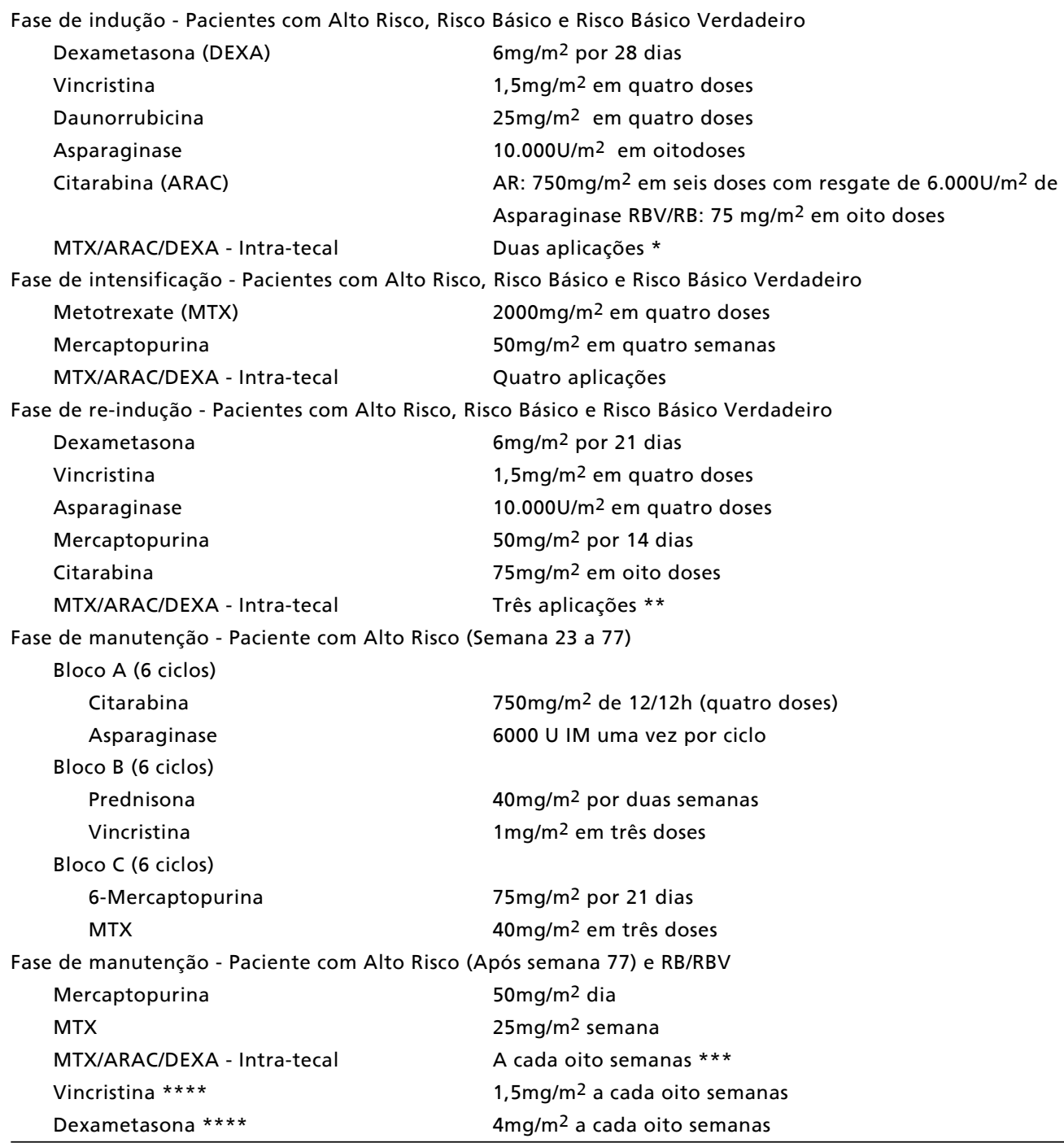

$A R=A l t o$ Risco; RB=Risco Básico; RBV=Risco Básico Verdadeiro * Quatro aplicações para $A R ;{ }^{*}$ *Cinco aplicações para $A R ; * * *$ Não realizado $A R ; * * * *$ Exclusivo para os pacientes de RB/AR.

Fonte: Sociedade Brasileira de Oncologia Pediátrica, Sociedade Brasileira de Hematologia e Hemoterapia. Protocolo Cooperativo GBTLI-LLA-93 para o Tratamento da Leucemia Linfóide Aguda na Infância. Campinas; 1993. 9

As variáveis analisadas foram: sexo, idade, principais sintomas e sinais, leucometria, imunofenótipo e grupo de risco ao diagnóstico, taxas de remissão, recaída, óbito e sobrevida global, local de recaída e variáveis de maior impacto para sobrevivência global.

Medidas descritivas foram usadas para análise estatística. O tempo de sobrevida dos pacientes foi estimado através da função de sobrevida de KaplanMeier e Log-Rank. O efeito de fatores de risco no tempo de sobrevida foi avaliado através do Modelo de Regressão de Cox. ${ }^{10}$ Os valores foram considerados estatisticamente significantes com $p \leq 0,05$.

O trabalho foi submetido e aprovado pelo Comitê de Ética da Fundação HEMOPE em conformidade com a resolução 196 da Comissão 
Nacional de Ética em Pesquisa do Ministério da Saúde.

\section{Resultados}

A proporção masculino: feminino encontrada foi de 1,7:1 e não houve diferença significante do ponto de vista estatístico quanto ao sexo em relação à sobrevida $(p=0,799)$.

A mediana de idade dos pacientes ao diagnóstico foi de oito anos e a casuística não apresentou pacientes com idade inferior a um ano. A sobrevida global em oito anos foi de $73 \%$ para os menores de 10 anos e de $49 \%$ para os de faixa etária superior, havendo diferença significativa quando avaliada pelo Teste Log-Rank ( $p=0,019)$.

Os principais sintomas e sinais clínicos presentes ao diagnóstico são apresentados na Tabela 2. Outras variáveis clínico-laboratoriais encontram-se na Tabela 3. A mediana da leucometria ao diagnóstico foi de 24.050 leucócitos $/ \mathrm{mm}^{3}$. A análise da curva de sobrevida mostrou diferença entre os três grupos categorizados quanto à leucometria ao diagnóstico $(p=0,001)$.

Todos os casos foram imunofenotipados e não houve diferença nas curvas de sobrevida global entre os pacientes com LLA de precursor B e T $(p=0,234)$. A presença do antígeno CD10 foi pesquisada em 105 casos e as curvas de sobrevida global não mostraram diferença estatisticamente significante entre os pacientes CD10 positivo e negativo $(p=0,201)$.

A taxa de remissão foi de $86 \%$ com $93 \%$ para o RBV, $96 \%$ para o RB e $82 \%$ do AR. Entre os 15 pacientes que não alcançaram remissão, $6(5,5 \%)$ corresponderam aos óbitos na indução. Ao final do estudo, dos 108 pacientes analisados $59 \%$ estavam vivos, $34 \%$ foram a óbito e $7 \%$ corresponderam às perdas de seguimento. A curva de sobrevida global da série foi de $62,5 \%$ após oito anos de seguimento. Houve diferença na sobrevida global entre os três grupos estudados $(\mathrm{p}=0,056)$ com valores de $74 \%$ para o grupo de RBV, $86 \%$ para o RB e $54 \%$ para o AR como mostra a Figura 1.

Quanto à frequiência de recaídas, foram estudados $73 \%$ dos pacientes, pois, os demais foram considerados não-avaliáveis quanto à essa variável por não terem alcançado remissão, terem ido a óbito em remissão ou serem perdas de seguimento. Entre os 79 casos avaliados, 19 recaíram o que corresponde a uma taxa de $24 \%$. As recaídas ocorreram de forma isolada na medula óssea $(63 \%)$ e no SNC (21\%), ou de forma combinada, em medula óssea/SNC (11\%) e medula óssea/testículo (5\%).

A análise multivariada, segundo modelo de Cox,${ }^{10}$ mostrou que as variáveis de maior impacto para a sobrevivência foram a faixa etária e a leucometria. Dessas, a leucometria foi à única variável que apresentou significância estatística (Tabela 4).

\section{Tabela 2}

Sinais e sintomas ao diagnóstico de 108 pacientes pediátricos com leucemia linfóide aguda. Fundação de Hematologia e Hemoterapia de Pernambuco (HEMOPE), Pernambuco, janeiro de 1993 a dezembro de 2001.

\begin{tabular}{lcc}
\hline & \multicolumn{2}{c}{ Pacientes } \\
\cline { 2 - 3 } Sinais e sintomas & $\mathrm{n}$ & $\%$ \\
\hline Febre & 94 & 87,0 \\
Hepatomegalia & 94 & 87,0 \\
Adenomegalia & 92 & 85,0 \\
Esplenomegalia & 89 & 82,0 \\
Sangramento & 72 & 67,0 \\
Queixas músculo-esqueléticas & 55 & 51,0 \\
Alargamento do mediastino & 18 & 17,0 \\
Infiltração do sistema nervoso central & 9 & 8,0 \\
\hline
\end{tabular}


Variáveis encontradas ao diagnóstico, freqüências relativas e absolutas, sobrevida global dos pacientes com leucemia linfóide aguda. Fundação de Hematologia e Hemoterapia de Pernambuco (HEMOPE), Pernambuco, janeiro de 1993 a dezembro de 2001.

\begin{tabular}{|c|c|c|c|c|}
\hline & & & Sobrevida global (oito anos)* & \\
\hline Variáveis & $\mathrm{n}$ & $\%$ & $\%$ & $p$ \\
\hline \multicolumn{5}{|l|}{ Sexo } \\
\hline Masculino & 67 & 62,0 & 64,0 & \multirow{2}{*}{0,799} \\
\hline Feminino & 41 & 38,0 & 60,0 & \\
\hline \multicolumn{5}{|l|}{ Faixa etária (anos) } \\
\hline$\geq 1 \mathrm{a}<10$ & 63 & 58,0 & 73,0 & \multirow{2}{*}{0,019} \\
\hline$\geq 10$ & 45 & 42,0 & 49,0 & \\
\hline \multicolumn{5}{|l|}{ Leucometria $\left(\mathrm{mm}^{3}\right)$} \\
\hline$<10000$ & 38 & 35,0 & 75,0 & \multirow{4}{*}{0,001} \\
\hline$\geq 10000$ a $<50000$ & 30 & 28,0 & 75,0 & \\
\hline$\geq 50000$ & 39 & 36,0 & 43,0 & \\
\hline Ignorada & 01 & 1,0 & & \\
\hline \multicolumn{5}{|l|}{ Imunofenótipo } \\
\hline LLA precursor $B$ & 88 & 81,0 & 65,0 & \multirow{2}{*}{0,234} \\
\hline LLA-T & 20 & 19,0 & 51,0 & \\
\hline \multicolumn{5}{|l|}{ Antígeno CD 10} \\
\hline Positivo & 76 & 70,0 & 66,0 & \multirow{3}{*}{0,201} \\
\hline Negativo & 29 & 27,0 & 56,0 & \\
\hline Não realizado & 03 & 3,0 & & \\
\hline \multicolumn{5}{|l|}{ Grupo de Risco } \\
\hline Risco Básico Verdadeiro & 13 & 12,0 & 74,0 & \multirow{3}{*}{0,056} \\
\hline Risco Básico & 23 & 21,0 & 86,0 & \\
\hline Alto Risco & 72 & 67,0 & 54,0 & \\
\hline
\end{tabular}

* Teste Log-Rank; LLA=Leucemia linfóide aguda.

\section{Figura 1}

Curva de sobrevida dos 108 pacientes com leucemia linfóide aguda, tratados pelo protocolo GBTLI-93, de acordo com os grupos de risco. Fundação de Hematologia e Hemoterapia de Pernambuco (HEMOPE), Pernambuco, janeiro de 1993 a dezembro de 2001.

\section{Curvas de Sobrevida}

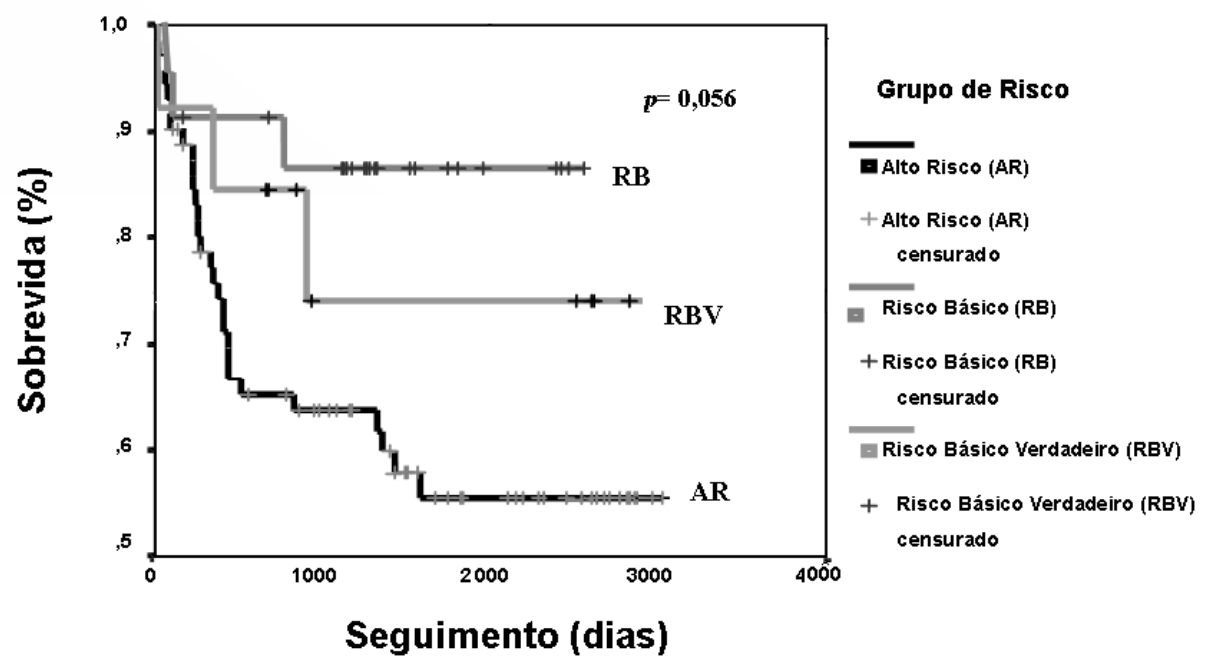


Tabela 4

Resultados do ajuste final do modelo de COX para 108 pacientes com leucemia linfóide aguda, tratados pelo protocolo GBTLI-93. Fundação de Hematologia e Hemoterapia de Pernambuco (HEMOPE), Pernambuco, janeiro de 1993 a dezembro de 2001

\begin{tabular}{lccc}
\hline Variável & Coeficiente & Valor de $\boldsymbol{p}$ & Risco relativo IC95\%* \\
\hline Faixa etária & 0,566 & 0,097 & $1,761(0,901-3,443)$ \\
Leucometria & 1,253 & $<0,001$ & $3,502(1,749-7,011)$ \\
\hline
\end{tabular}

* IC95\%=Intervalo de confiança 95\%.

\section{Discussão}

No presente estudo, a razão sexo masculino/feminino foi 1,7. Nos resultados do protocolo GBTLI-93 esta relação foi de $1,0 .{ }^{11}$ Por sua vez, uma pesquisa realizada na Bahia mostrou uma razão masculino:feminino de $1,4 .{ }^{12}$ Os resultados de largas séries internacionais confirmam que há discreto predomínio desta doença no sexo masculino. ${ }^{13-15}$ Em relação à sobrevivência não se observou diferença significante quando comparados pacientes masculinos e femininos. A variável sexo como fator de relevância prognóstica é controversa porém os mais reconhecidos grupos internacionais de tratamento não consideram essa variável na estratificação dos grupos de risco. ${ }^{13,14,16}$

A idade é considerada como fator de importância prognóstica independente em crianças com LLA. ${ }^{17-19}$ Pacientes com idade superior a nove anos apresentam pior prognóstico, o que fez com que esta variável e a leucometria ao diagnóstico tenham sido consideradas na estratificação de risco para LLA de precursor B. ${ }^{16}$ $\mathrm{Na}$ análise do estudo GBTLI-93 com 853 pacientes analisados foi encontrada a seguinte distribuição quanto à idade: $2 \%$ menores de um ano, $75 \%$ entre um e 10 anos e $23 \%$ entre 10 e 18 anos. ${ }^{11}$ A distribuição dos pacientes do presente estudo quanto à idade mostrou que $42 \%$ apresentavam idade maior ou igual a 10 anos, contribuindo para o elevado percentual de pacientes classificados como de alto risco. Esse fato pode não refletir a distribuição dessa variável na população de pacientes do Estado de Pernambuco e corresponder a um viés de seleção relacionado à casuística do serviço, onde o tratamento não é oferecido a crianças menores de um ano. Os pacientes com idade entre um e 10 anos apresentaram uma sobrevida global de $73 \%$ em oito anos, sendo esse resultado semelhante àqueles apresentados pelo GBTLI-93, nos quais a Sobrevida Livre de Eventos (SLE) foi de 73\% em seis anos. Nos pacientes com idade $\geq 10$ anos a sobrevida global em oito anos foi de $49 \%$ comparados a SLE 59\% do GBTLI-93. ${ }^{11}$

Quanto à freqüência dos sintomas e sinais ao diagnóstico, destacaram-se os resultados referentes às queixas músculo-esqueléticas e ao SNC. Essas queixas presentes em $51 \%$ dos pacientes foram representadas por artrites, artralgias e dores ósseas, o que pode ter dificultado o diagnóstico e a condução de pacientes pediátricos com LLA. Isso porque outros diagnósticos precisam ser considerados, tais como a dor de crescimento, artrite reumatóide juvenil, febre reumática, lúpus eritematoso sistêmico, osteomielite e anemia falciforme. Barbosa et al. ${ }^{20}$ em estudo realizado com 61 crianças portadoras de leucemia aguda, mostraram que $62 \%$ apresentavam manifestações músculo-esqueléticas. Dessas, sete tiveram diagnóstico inicial de dor benigna, calazar, lúpus eritematoso sistêmico, artrite reumatóide juvenil ou púrpura trombocitopênica imune e três pacientes receberam corticoterapia antes do diagnóstico definitivo. Assim, a suspeita de leucemia deve ser considerada no diagnóstico diferencial de pacientes pediátricos com queixas músculo-esqueléticas. Esses autores ${ }^{20}$ alertaram também para o fato de que as queixas músculoesqueléticas são comuns nos pacientes portadores de leucemias agudas, embora os exames iniciais possam ser normais ou com alterações discretas que não apontam para o diagnóstico da neoplasia. Além disso, o uso de corticosteróides pode aliviar os sintomas, alterar a citologia e histologia medular retardando o diagnóstico fazendo com que o paciente chegue ao centro de referência com uma doença avançada e resistente.

A freqüência de infiltração do SNC, de $8 \%$, encontrada no presente estudo, foi superior aos resultados nacionais utilizando o GBTLI-93, que atingiram percentuais de $1,7 \% .^{11} \mathrm{Na}$ Bahia, os resultados da avaliação de pacientes tratados pelos protocolos GBTLI-85 e GBTLI-93, revelaram freqüências de $3,5 \%$ e $6,7 \%$, respectivamente. ${ }^{12}$ Estudos em outros 
países relatam uma freqüência que varia de $0,6 \%$ a $12,4 \% .^{13,14}$ Essas discrepâncias podem ser secundárias à dificuldade neste diagnóstico o que reforça a necessidade de câmaras técnicas associadas aos protocolos para análise dos casos de difícil definição.

De acordo com o Consenso Internacional, publicado em 1996, pacientes com LLA de precursor B com menos de 50.000 leucócitos $/ \mathrm{mm}^{3}$ ao diagnóstico, por apresentarem melhor prognóstico, devem ser incluídos dentro do grupo de Risco Básico, enquanto que aqueles com leucometria superior são classificados como Alto Risco e, portanto, devem ser submetidos a tratamento mais intensivo. ${ }^{16}$ Os dados brasileiros sobre a leucometria inicial em crianças com LLA mostram que $26 \%$ dos pacientes incluídos no estudo GBTLI-93 e 36,1\% dos pacientes da Bahia apresentavam leucometria > que 50.000/. $\mathrm{mm}^{3} .{ }^{11,12}$

No presente trabalho, $37 \%$ dos pacientes apresentaram mais que $50.000 / \mathrm{mm}^{3}$ leucócitos, sendo esse resultado semelhante ao trabalho desenvolvido na Bahia, porém, superior em relação aos resultados do GBTLI-93. ${ }^{11}$ Estudos internacionais mostram que menos de $20 \%$ dos casos de LLA possuem mais de $50.000 / \mathrm{mm}^{3}$ leucócitos ao diagnóstico. ${ }^{13,14}$ Deve ser enfatizada a importância da leucometria para a estimativa global da sobrevida dos pacientes. De fato, a leucometria foi o único fator de impacto prognóstico encontrado através da análise multivariada.

A freqüência do fenótipo $\mathrm{T}$ nos pacientes com LLA da infância varia entre $7,4 \%$ a $16,4 \%$, em estudos nacionais e internacionais. ${ }^{11,13,14,21,22}$ A presença desse fenótipo no presente estudo foi de $19 \%$, o que é um provável reflexo do elevado número de pacientes maiores de 10 anos de idade.

As curvas de sobrevivência global não indicaram diferenças significativas quando comparados os pacientes com LLA de precursor B e LLA-T, bem como entre aqueles que expressavam o antígeno CD10 positivo ou negativo. Esse resultado difere do GBTLI-93 onde os pacientes com LLA de precursor B apresentaram uma sobrevida livre de eventos (SLE) em seis anos de aproximadamente $72 \%$ e na LLA-T de $44 \% .{ }^{11}$ Ainda segundo esses dados, pacientes com fenótipo CD10 positivos apresentaram uma SLE em seis anos em torno de $74 \%$, contra $34 \%$ dos casos negativos. Neste estudo, o fenótipo T continuou tendo impacto prognóstico na análise multivariada.

A importância prognóstica do imunofenótipo das células blásticas foi relatada na literatura, entretanto a importância dessa variável tem diminuído com os avanços no tratamento. ${ }^{23}$ Um Grupo italiano ${ }^{13}$ estudou 632 crianças com LLA mostrando que, apesar da análise univariada encontrar melhor sobrevida nos pacientes com LLA de precursor B, essa variável perdeu o impacto no estudo multivariado. O Instituto de Câncer Dana-Farber publicou dados de 1225 crianças tratadas para LLA, das quais 125 apresentavam imunofenótipo $\mathrm{T}$, no entanto, não foram demonstradas diferenças na sobrevida entre os pacientes com esse imunofenótipo em relação aqueles com LLA de precursor $\mathrm{B}^{24}$

O protocolo GBTLI-93 preconiza três grupos de risco ( $R B V, R B$ e $A R$ ), sendo que $48 \%$ dos pacientes foram classificados como AR, $34 \% \mathrm{RB}$ e $18 \% \mathrm{RBV}^{11}$ $\mathrm{Na} \mathrm{Bahia}^{12}{ }^{2} 57,5 \%$ foram considerados AR e $42,5 \%$ $\mathrm{RB}$, sendo que este grupo incluiu também os pacientes classificados com RBV pelo GBTLI-93. No HEMOPE, 67\% dos pacientes estavam incluídos no grupo de AR, $21 \%$ no de RB e $12 \%$ no RBV. Esses dados da Bahia e do HEMOPE revelam um maior número de pacientes de alto risco quando comparados com os resultados do estudo nacional GBTLI-93. ${ }^{11}$

O percentual de remissão obtido $(86 \%)$ é baixo quando comparado aos resultados obtidos com o uso do GBTLI-93 e a estudos internacionais, os quais mostram valores superiores a 95\%. ${ }^{11,13-15}$ Os óbitos na indução foram incluídos entre aqueles que não alcançaram remissão e corresponderam a $5,5 \%$ da população estudada. Nos resultados do GBTLI-93 ${ }^{11}$ esta freqüência foi de $3 \%$ e em estudos internacionais o percentual de óbitos nessa fase variou de $0,5 \%$ a $1 \% .^{13,14,24}$ Conclui-se, portanto, que os óbitos na indução no Brasil ainda são considerados altos quando comparados com os resultados de protocolos de tratamento internacionais, tais como o DanaFarber $^{14,24}$ e o AIEOP-ALL 87. ${ }^{13}$

Quanto à recaída, foi possível avaliar 79 pacientes e desses 19 (24\%) recaíram, mostrando que essa taxa também foi elevada quando comparada com os dados do GBTLI-93 onde $13,4 \%$ de 817 pacientes recaíram. $^{11}$

A Sobrevida Global (SG) para todos os pacientes desta série foi de $62,5 \%$ para um período de seguimento de oito anos. No GBTLI-93 esse valor correspondeu a $70 \%$ para um período de seguimento de seis anos. ${ }^{11}$ A sobrevida dos pacientes tratados na Fundação HEMOPE variou de acordo com o grupo de risco: $74 \%$ para RBV, $86 \%$ para $\mathrm{RB}$ e $54 \%$ para AR. No GBTLI-93 a sobrevida foi de $84 \%$ para RBV, $77 \%$ para o RB e $58 \%$ para AR. ${ }^{11}$ Os resultados da presente investigação são diferentes dos resultados nacionais que mostraram melhor sobrevida para o RBV. ${ }^{11} \mathrm{O}$ número limitado de pacientes incluídos no RBV neste trabalho não permite contudo conclusões definitivas que explique o motivo dessa diferença.

Um estudo publicado na região sul do Brasil utilizando os protocolos do grupo Berlim-Frankfurt- 
Munique versões 90 e 95 (BFM 90 e 95) mostrou uma SG de $57 \%$ e sobrevida livre de eventos de $50,8 \%$ em cinco anos. ${ }^{25} \mathrm{Um}$ grupo argentino usou o protocolo BFM 90 com pequenas modificações em 885 pacientes e obteve uma taxa de SLE de $64 \% .{ }^{26}$ A SG e a SLE em 10 anos do protocolo italiano foi de $74,7 \%$ e $62,8 \%$, respectivamente. ${ }^{13}$ Os dados americanos do Instituto de Câncer Dana-Farber mostram uma SLE em cinco anos de $83 \% .{ }^{14}$ Os protocolos de Hong Kong 93 e 97 (HKALL 93 e 97) indicaram SG em quatro anos de $81,8 \%$ e $86,5 \%$ respectivamente, com SLE de $65 \%$ e $79 \%{ }^{15}$

A análise global dos resultados do presente estudo mostrou menor taxa de remissão e sobrevida global, maiores percentuais de recaída e taxa de óbitos na indução, comparativamente ao GBTLI-93. ${ }^{11}$ Esses

\section{Referências}

1. Kebriaei P, Anastasi J, Larson, RA. Acute lymphoblastic leukemia: diagnosis and classification. Best Pract Res Clin Haematol. 2003; 15: 597-21.

2. Mauer AM. Acute lymphocytic leukemia. In: Beutler E, Lichtman MA, Coller, MB. Williams Hematology. New York: McGraw-Hill; 1995. p.1004-16.

3. Farhi DC, Rosenthal NS. Acute lymphoblastic leukemia. Clin Lab Med. 2000; 20: 17-28.

4. Zanrosso CW, Hatagima A, Emerenciano M, Figueiredo FRA, Félix TM, Segal SL, Guigliani R, Muniz MTC, Oliveira MS. The role of methylenetetrahydrofolate reductase in acute lymphoblastic leukemia in a Brazilian mixed population. Leuk Res. 2005; 30: 477-81.

5. Groves FD, Linet MS, Devesa SS. Patterns of occurrence of the leukaemias. Eur J Cancer. 1995; 31: 941-9.

6. Oliveira MS, Cordoba JC, Alencar DM, Campos MM, Carriço K, Dobbin J, Dorea MD, Ferreira R, Mendonça N, Magalhães IS. Biological diversity variations of pediatric acute leukemia in Brazil: contribution of immunophenotypic profiles to epidemiological studies. Rev Bras Hematol Hemoter. 2005; 27: 21-6.

7. Paes CA, Viana MB, Freire RV, Martins-Filho AO, Taboada DC, Rocha VC. Direct association of socio-economic status with T-cell acute lymphoblastic leukaemia in children. Leuk Res. 2003; 27: 789-94.

8. Vila PB, Garcia CP, Riera MS, Aramburu JJ, Cordina JS. Leucemia mínima residual: nuevo concepto de remisión completa. An Pediatr. (Barc., Spain) 2005; 63: 390-5.

9. Sociedade Brasileira de Oncologia Pediátrica. Sociedade Brasileira de Hematologia e Hemoterapia. Protocolo Cooperativo GBTLI-LLA-93 para o Tratamento da Leucemia Linfóide Aguda na Infância. Campinas; 1993.

10. Cox DR. Regression models and life tables. J Royal Stat Soc Ser B Methodol. 1972; 34: 187-95. fatos podem ser decorrentes do maior percentual de pacientes de alto risco da série estudada. Por outro lado, a única variável de impacto independente foi a leucometria, em concordância com a literatura que também tem evidenciado a importância desta variável entre aquelas de impacto na sobrevida.

Os resultados do tratamento em crianças com LLA podem ser o reflexo das condições de assistência à saúde, situação socioeconômica e/ou fatores genéticos associados. É importante que novos estudos sejam realizados no intuito de avaliar o impacto dessas variáveis no resultado do tratamento, uma vez que há relato na literatura de que crianças de países em desenvolvimento, quando expostas às condições ideais de tratamento, apresentam resultados semelhantes àqueles de países desenvolvidos. ${ }^{27}$
11. Sociedade Brasileira de Oncologia Pediátrica. Protocolo de Tratamento da Leucemia Linfoblástica Aguda na InfânciaGBTLI-LLA-1999. Campinas; 2000.

12. Barreto LHS. Estudo de sobrevida em crianças portadoras de leucemia linfoblástica aguda. [dissertação mestrado]. Salvador: Universidade Federal da Bahia; 2001.

13. Paolucci G, Vecchi V, Favre C, Miniero R, Madon E, Pession A, Rondelli R, Rossi G, Lo Nigro L, Porta F, Santoro N, Indolfi P, Basso G, Conter V, Arico M. Treatment of childhood acute lymphoblastic leukemia. Long-term results of the AIEOP-ALL 87 study. Haematology. 2001; 86: 478-84.

14. Silverman LB, Gelber RD, Dalton VK, Asselin BL, Barr RD, Clavell LA, Urwitz CA, Moghrabi A, Samson Y, Schorin MA, Arkin S, Declerck L, Cohen HJ, Sallan SE. Improved outcome for children with acute lymphoblastic leukemia: results of Dana-Farber Consortium Protocol 9101. Blood. 2001; 97: 1211-8.

15. Li CR, Chik KW, Ha SY, Lee ACW, Yuen HL, Ling SC, Lee V, Chan GCF, Shing MMK, Chan LC, Ng MHL. Improved outcome of acute lymphoblastic leukaemia treated by delayed intensification in Hong Kong children: HKALL 97 study. Hong Kong Med J. 2006; 12: 33-9.

16. Smith M, Arthur D, Camitta B, Carroll AJ, Crist W, Gaynon P, Gelber R, Heerema N, Korn EL, Link M, Murphy S, Pui CH, Pullen J, Reamon G, Sallan SE, Sather H, Shuster J, Simon R, Trigg M, Tubergen D, Uckun F, Ungerleider R. Uniform approach to risk classification and treatment assignment for children with acute lymphoblastic leukemia. J Clin Oncol. 1996; 14: 4-6.

17. Sather H. Age at diagnosis in childhood acute lymphoblastic leukemia. Med Pediatr Oncol. 1986; 14: 166-72. 
18. Crist W, Boyett J, Pullen J, van Eys J, Vietti T. Clinical and biological features predict poor prognosis in acute lymphoid leukemia in children and adolescents: a Pediatric Oncology Group Review. Med Pediatr Oncol. 1986; 14: $135-9$.

19. Crist W, Shuster J, Look T, Borowitz M, Behm F, Bowman P, Frankel L, Pullen J, Krance R, Steuber P. Current results of studies of imunophenotype. Age and leukocyte-based therapy for children with acute lymphoblastic leukemia. Leukemia. 1992; 6: 162-6.

20. Barbosa CMP, Nakamura C, Terreri MT, Lee MLM, Petrilli AS, Hilário MOE. Manifestações músculo-esqueléticas como apresentação inicial das leucemias agudas na infância. J Pediatr. (Rio J). 2002; 78: 481-4.

21. Rego EM, Garcia AB, Viana SR, Falcao RP Characterization of acute lymphoblastic leukemia subtypes in Brazilian patients. Leuk Res. 1996; 20: 349-55.

22. Silva ML, Ornellas de Souza MH, Ribeiro RC, Land MG Boulhosa de Azevedo AM, Vasconcelos F, Otero L, Vasconcelos Z, Bouzas LF, Abdelhay E. Cytogenetic analysis of 100 consecutives newly diagnosed cases of ALL in Rio de Janeiro Brazilian children. Cancer Genet Cytogenet. 2002; 137: 85-90.

Recebido em 10 de dezembro de 2006

Versão final apresentada em 17 de junho de 2007

Aprovado em 31 de julho de 2007
23. Carroll WL, Bhojwani D, Min DJ, Raetz E, Relling M, Davies S, Downing JR, Willman CL, Reed JC. Pediatric acute lymphoblastic leukemia. Hematology (Am Soc Hemaol Educ Program) 2003; 50: 102-31

24. Goldberg JM, Silverman LB, Levy DE, Dalton VK, Gelber RD, Lehmann L, Cohen HJ, Sallan SE, Asselin BL. Childhood T-cell acute lymphoblastic leukemia: the DanaFarber Cancer Institute Acute Lymphoblastic Leukemia Consortium Experience. J Clin Oncol. 2003; 21: 3616-22.

25. Laks D, Longhi F, Wagner MB, Garcia PCR. Avaliação da sobrevida de crianças tratadas com leucemia linfocítica aguda tratadas pelo protocolo Berlim-Frankfurt-Munique. J Pediatr. 2003; 79: 149-58

26. Sackmann-Muriel F, Felice MS, Zubizarreta PA, Alfaro E, Gallego M, Rossi J, Cygler AM. Treatment results in childhood acute lymphoblastic leukemia with a modified ALLBFM 90 protocol: lack of improvement in high-risk group. Leuk Res. 1999; 23: 331-40.

27. Brandalise SR. Acute lymphoblastic leukemia in children. Arch Med Intern. 2007; Supl 1; 29: S92-S5. 